\title{
Adjoint inversion of the thermal structure of Southeastern Australia
}

\author{
B. Mather ${ }^{\oplus},{ }^{1}$ L. Moresi ${ }^{2,3}$ and P. Rayner ${ }^{2}$ \\ ${ }^{1}$ School of Geoscience, The University of Sydney, Sydney, NSW 2006, Australia. E-mail: brmather1@gmail.com \\ ${ }^{2}$ School of Earth Sciences, The University of Melbourne, Melbourne, VIC 3010, Australia \\ ${ }^{3}$ Research School of Earth Sciences, The Australian National University, Canberra, ACT 0200, Australia
}

Accepted 2019 August 8. Received 2019 July 28; in original form 2019 March 7

\begin{abstract}
SUMMAR Y
The variation of temperature in the crust is difficult to quantify due to the sparsity of surface heat flow observations and lack of measurements on the thermal properties of rocks at depth. We examine the degree to which the thermal structure of the crust can be constrained from the Curie depth and surface heat flow data in Southeastern Australia. We cast the inverse problem of heat conduction within a Bayesian framework and derive its adjoint so that we can efficiently find the optimal model that best reproduces the data and prior information on the thermal properties of the crust. Efficiency gains obtained from the adjoint method facilitate a detailed exploration of thermal structure in SE Australia, where we predict high temperatures within Precambrian rocks of $6500^{\circ} \mathrm{C}$ due to relatively high rates of heat production $\left(0.9-1.4 \mu \mathrm{W} \mathrm{m}^{-3}\right)$. In contrast, temperatures within dominantly Phanerozoic crust reach only $520{ }^{\circ} \mathrm{C}$ at the Moho due to the low rates of heat production in Cambrian mafic volcanics. A combination of the Curie depth and heat flow data is required to constrain the uncertainty of lower crustal temperatures to $\pm 73^{\circ} \mathrm{C}$. We also show that parts of the crust are unconstrained if either data set is omitted from the inversion.
\end{abstract}

Key words: Composition and structure of the continental crust; Heat flow; Australia; Inverse theory; Joint inversion; Numerical modelling; Heat generation and transport; Rheology: crust and lithosphere.

\section{INTRODUCTION}

Understanding the variation of temperature in the Earth's crust is fundamental in evaluating the geothermal potential of a region and its mechanical stability. However, sparse heat flow data and limited measurements of the thermal properties of rocks confound attempts to model the thermal structure of the crust. Crustal geotherms are controlled by thermal conductivity and rates of heat production. In many cases, heterogeneity in these physical properties may be represented by the values typical of their lithology and treated as prior geological information. Their values remain inherently uncertain and small deviations from the reference values can result in significantly altered temperature profiles. Fortunately, there are multiple direct and indirect observations on the temperature field that can restrict the range of acceptable models.

Multiple geophysical methods have been proposed to resolve the thermal structure of the lithosphere with varying degrees of precision. Geotherms may be constructed from xenolith data (O'Reilly \& Griffin 1985; Cull et al. 1991), but the spatial coverage of xenoliths are often limited. Seismic velocity has proven effective to infer upper-mantle temperature (e.g. Goes et al. 2005), but its application relies on building a compositional model suitable for the geological context and estimating attenuation from grain size and water content
(Cammarano et al. 2003; Karato 2003; Faul \& Jackson 2005). In this paper we incorporate two independent observations: the Curie depth, which is interpreted to be the $580{ }^{\circ} \mathrm{C}$ isotherm, and surface heat flow, which records integrated information on the Earth's heat flow budget. We formally constrain the variation of thermal structure by adopting a Bayesian approach that jointly assimilates these data and a priori information on the spatial variation of thermal conductivity and heat production.

A common obstacle in the Bayesian inversion is the large number of simulations required to adequately sample the posterior density function. For models of thermal structure, this involves solving temperature with prescribed boundary conditions across a mesh populated with constitutive properties, such as thermal conductivity and heat production. There is an inherent trade-off in computational cost between the number of models in an ensemble and their complexity/resolution, which can be a significant disadvantage for any Monte-Carlo-based inversion when the forward model is irreducibly expensive to compute. By contrast, gradient-based optimization requires substantially fewer evaluations than Monte Carlo techniques to arrive at an optimal solution.

We formulate the gradient analytically for the inverse problem of heat conduction so we can efficiently estimate temperature distributions in the crust, subject to a trade-off between data and prior 


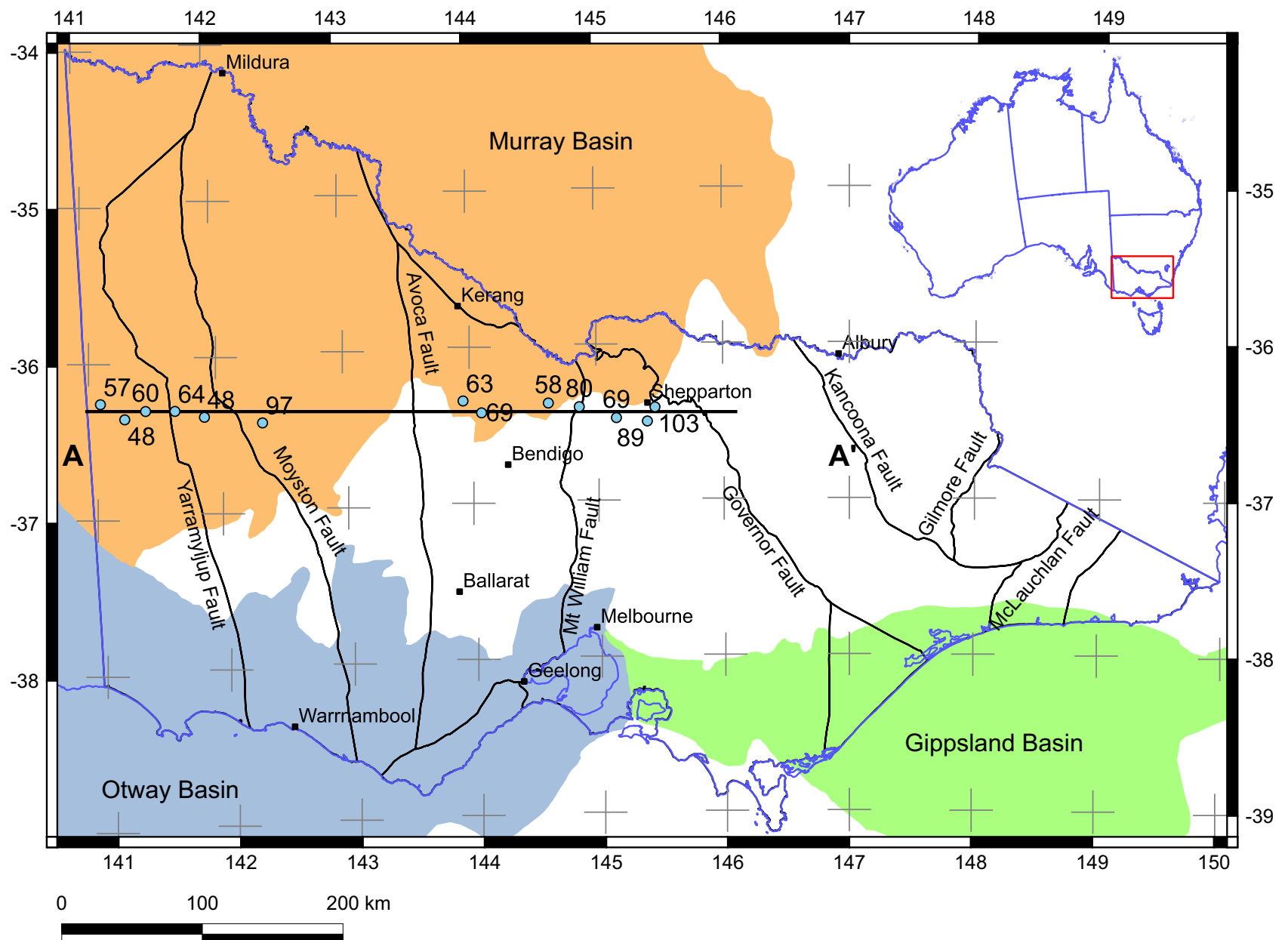

Figure 1. Major structures and sedimentary basins in Southeast Australia. A to A' indicates the 2-D cross-section we use as a case study for the adjoint solver. Heat flow observations along the cross-section plane are indicated by circles with their values adjacent in $\mathrm{mW} \mathrm{m}^{-2}$.

information, without sacrificing resolution. Our mathematical formulation of the gradient is general: it does not depend on the number of dimensions and it can be applied to any study area. We specifically apply it to invert the thermal structure of Southeastern Australia where well-constrained prior geological information already exists, there is a high density of heat flow estimates, and maps of the Curie depth are readily available.

\subsection{Geological structure}

We use geological maps to provide prior estimates of the thermal conductivity and heat production within our model domain. Geological information preserves sharp geological boundaries that are known to exist and simplifies the number of variables to invert. In this case, values of thermal conductivity and heat production are mapped to discreet regions of the crust defined as a 'lithology', where the number of unknowns corresponds to the number of lithologies within the study area. Fewer inversion variables are computationally easier to invert and avoid non-unique solutions in the alternative case where no prior geological information exists. In SE Australia, the spatial variation of geological layers is well-resolved by structural information, seismic reflections, magnetic and gravity data that have been integrated within a geological model of the region (Rawling et al. 2011). The geological model resolves a sharp boundary from dominantly Proterozoic to Phanerozoic crust, delineated by the Moyston Fault (Fig. 1), and divides the Delamerian and Lachlan heat flow provinces (Mather et al. 2017). Assuming a mechanically stable crust that is in thermal steady state, the inverse methodology we formulate estimates the optimal configuration of lithology-dependent thermal conductivity and heat production that best reproduce these data. From here we estimate the uncertainty and covariance relationships within the parameter space and the degree to which subsurface temperatures can vary.

\section{THE INVERSE PROBLEM OF HEAT CONDUCTION}

In a Bayesian framework, information on input parameters is represented in probabilistic terms (e.g. Mosegaard \& Tarantola 1995). The solution is given by a posteriori probability, $P(\mathbf{m} \mid \mathbf{d})$, which describes the probability of a particular model, $\mathbf{m}$, given the data, d,

$P(\mathbf{m} \mid \mathbf{d}) \propto P(\mathbf{d} \mid \mathbf{m}) \cdot P(\mathbf{m})$,

where the likelihood function, $P(\mathbf{d} \mid \mathbf{m})$, is the probability of reproducing the data given a particular model, and the a priori probability, $P(\mathbf{m})$, is what we know about the model before assimilating the data. For a wide class of probability density functions, the posterior probability can be evaluated through an objective function, $S(\mathbf{m})$, 
which jointly compares the misfit of the model to data and prior information, $\mathbf{m}_{p}$,

$P(\mathbf{m} \mid \mathbf{d})=A \exp (-S(\mathbf{m}))$,

where $A$ is a constant. We seek the maximum a posteriori (MAP) estimate, which may be obtained by minimizing the nonlinear leastsquares objective function for Gaussian distributions, after Tarantola (2005),

$$
\begin{aligned}
S(\mathbf{m})= & \frac{1}{2}\left[(\mathbf{g}(\mathbf{m})-\mathbf{d})^{\mathrm{T}} \mathbf{C}_{d}^{-1}(\mathbf{g}(\mathbf{m})-\mathbf{d})\right. \\
& \left.+\left(\mathbf{m}-\mathbf{m}_{p}\right)^{\mathrm{T}} \mathbf{C}_{p}^{-1}\left(\mathbf{m}-\mathbf{m}_{p}\right)\right],
\end{aligned}
$$

where $\mathbf{C}_{d}$ and $\mathbf{C}_{p}$ are the data and prior covariance matrices, respectively. Eq. (3) simplifies to the $\ell_{2}$-norm objective function if data and prior information are uncorrelated,

$S(\mathbf{m})=\frac{1}{2} \sum_{i} \frac{\left|g^{i}(\mathbf{m})-d^{i}\right|^{2}}{\left(\sigma_{d}^{i}\right)^{2}}+\frac{1}{2} \sum_{j} \frac{\left|\mathrm{m}^{j}-\mathrm{m}_{p}^{j}\right|^{2}}{\left(\sigma_{p}^{j}\right)^{2}}$,

where $\mathbf{m}$ is the model and $\mathbf{g}(\mathbf{m})$ is the forward operator, which is the prediction of observations from the model. For our exploration of thermal structure, $\mathbf{g}(\mathbf{m})$ describes the mapping of thermal conductivity and heat production to a mesh over which the steady-state heat equation is solved with prescribed boundary conditions. The calculation of surface heat flux and extraction of the Curie isotherm from the temperature simulation establishes an explicit link to data, d. The second term in eq. (4) compares $\mathbf{m}$ with a priori information on thermal conductivity, $k$, and heat production, $H$, obtained from measurements on rock samples from different lithologies.

\subsection{Forward model}

The input vector $\mathbf{m}$ in our forward problem comprises values of thermal conductivity $(k)$ and heat production $(H)$ for each lithology, described by our prior geological model, and a uniform lower flux boundary condition, $q_{0}$. The input vector is thus $\mathbf{m}:\left[k_{1}, k_{2}, \ldots, k_{n}, H_{1}, H_{2}, \ldots, H_{n}, q_{0}\right]$ where $n$ is the number of lithologies.

First, we map the lithology-dependent parameters $(k$ and $H)$ to a regularly spaced Cartesian mesh, $f: \mathbf{m}\left(L_{i}\right) \rightarrow \Omega$, where $f$ is the function that maps the thermal properties to a region of the mesh $\Omega$ occupied by a given lithology $L_{i}$. We solve the steady-state heat equation,

$\nabla(\mathbf{k} \nabla \mathbf{T})=-\mathbf{H}$

with insulated side boundary conditions, a Neumann lower boundary condition $\left(q_{0}\right)$, and a Dirichlet upper boundary condition corresponding to mean annual surface temperature. We implement a temperature-dependent conductivity term, $k(T)$, using the parametrization of Hofmeister (1999),

$\mathbf{k}(\mathbf{T})=k_{0}\left(\frac{298}{\mathbf{T}}\right)^{a}, \quad 0 \leq a \leq 1$

where $k_{0}$ is the conductivity measured at room temperature, and $a$ controls the dependence of conductivity on temperature. A temperature solution can be obtained from the solution of linear equations,

$\mathbf{A T}=\mathbf{b}$,

where $\mathbf{A}$ is a banded square matrix containing second-order finite difference equations, and $\mathbf{b}$ is a vector that contains the heat source and boundary condition parameters. Since $k$ depends on temperature, eq. (5) is nonlinear and we must iteratively solve for temperature using successive updates to $\mathbf{A}$ until the difference in solutions approach zero (we accept a solution when $\left|\mathbf{T}_{n}-\mathbf{T}_{n-1}\right|<1 \times 10^{-8}$ ). The nonlinearity in $k$ decreases as $a \rightarrow 0$ and $k(T) \rightarrow k_{0}$.

The temperature solution is compared to our Curie depth and heat flow data in the objective function. Expanding eq. (3) we obtain,

$$
\begin{aligned}
2 S\left(\mathbf{k}, \mathbf{H}, q_{0}\right)= & \left(z_{T_{580}}\left(\mathbf{k}, \mathbf{H}, q_{0}\right)-z_{T_{580}}\right) \\
& \times \mathbf{C} z_{T_{580}}^{-1}\left(z_{T_{580}}\left(\mathbf{k}, \mathbf{H}, q_{0}\right)-z_{T_{580}}\right) \\
& +\frac{\left(q_{s}\left(\mathbf{k}, \mathbf{H}, q_{0}\right)-q_{s}\right)^{2}}{\sigma_{q_{s}}^{2}}+\frac{\left(\mathbf{k}-\mathbf{k}_{p}\right)^{2}}{\sigma_{\mathbf{k}}^{2}} \\
& +\frac{\left(\mathbf{H}-\mathbf{H}_{p}\right)^{2}}{\sigma_{\mathbf{H}}^{2}}+\frac{\left(q_{0}-q_{0_{p}}\right)^{2}}{\sigma_{q_{0}}^{2}},
\end{aligned}
$$

where $z_{T_{580}}$ is the depth to the $580{ }^{\circ} \mathrm{C}$ isotherm, and $q_{s}$ is the heat flux at the surface of the model. The $\ell_{2}$-norm is used for $q_{s}$, because heat flow data are all uncorrelated observations of the thermal field, and the nonlinear least-squares objective function is used for the Curie depth.

\subsection{Gradient methods}

The cost of finding the MAP estimate from exhaustive evaluations of the forward model is prohibitive. Instead, gradient-based inversion techniques use the derivative of the forward model $\nabla S(\mathbf{m})$ to efficiently find the minimum of $S(\mathbf{m})$. The gradient-descent method for finding successively better approximations to a minimum of a function, $S(\mathbf{m})$, is given as

$\mathbf{m}_{i+1}=\mathbf{m}_{i}-\mu \nabla S\left(\mathbf{m}_{i}\right)$,

where $\nabla S\left(\mathbf{m}_{i}\right)=\mathbf{J}\left(\mathbf{m}_{i}\right)^{T} \mathbf{g}\left(\mathbf{m}_{i}\right)$ describes the relationship with the Jacobian $\mathbf{J}(\mathbf{m})$, and $\mu$ is a line search parameter to control step size. Evaluating $\nabla S(\mathbf{m})$ analytically is key to the most efficient gradientbased optimization and motivates the use of an adjoint formulation.

\subsection{Adjoint model}

The first step in any adjoint approach is to backward-propagate derivatives of the objective function with respect to the forward operator $\mathbf{g}(\mathbf{m})$ and model parameters $\mathbf{m}$ through the forward model. This leads to the following expression:

$\mathrm{d} \mathbf{g}(\mathbf{m})+\mathrm{d} \mathbf{m}=\mathbf{C}_{d}^{-1}(\mathbf{g}(\mathbf{m})-\mathbf{d})+\mathbf{C}_{p}^{-1}\left(\mathbf{m}-\mathbf{m}_{p}\right)$,

relating the data and prior terms in eq. (3), respectively. The next steps require the differentiation of the forward operator with respect to model parameters in order to find the gradient, dm. We have already obtained the derivative of the objective function with respect to a priori information, from the second term in eq. (10), which is summed at the end.

The derivatives of the temperature solution with respect to matrix $\mathbf{A}$ and vector $\mathbf{b}$ are

$\mathrm{d} \mathbf{T}=\mathbf{A}^{-1} \mathrm{~d} \mathbf{b}+\mathrm{d} \mathbf{A}^{-1} \mathbf{b}$,

$\mathbf{A}$ and $\mathbf{b}$ are already obtained from the forward model, and their derivatives with respect to model parameters are found by applying the chain rule. Vector $\mathbf{b}$ represents heat sources, $\mathbf{H}$, and the lower Neumann boundary condition, $q_{0}$. The adjoint is found by evaluating,

$\mathrm{d} \mathbf{b}=\left(\mathbf{A}^{-1}\right)^{\mathrm{T}} \mathrm{d} \mathbf{T}$ 


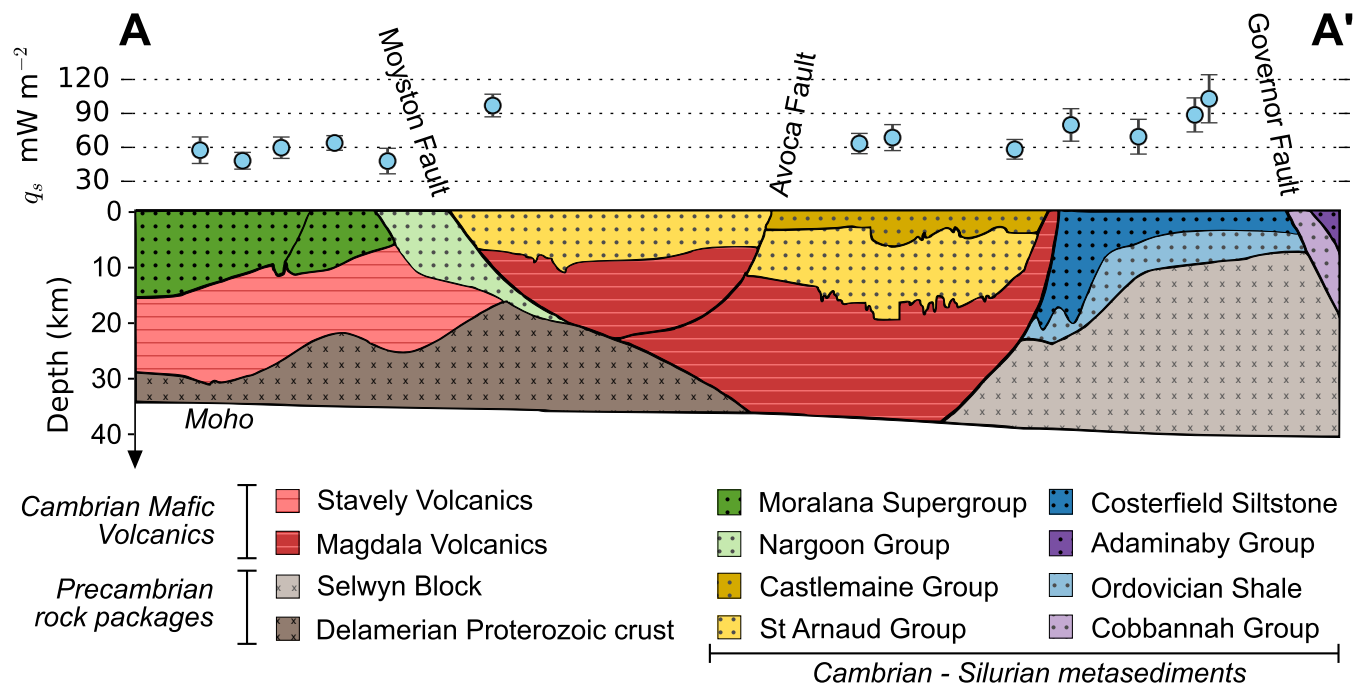

Figure 2. Simplified cross-section between $\mathbf{A}$ and $\mathbf{A}^{\prime}$. Each major lithology is indicated by a distinct colour. The distribution of surface heat flow observations along the plane is shown above with error bars indicating one standard deviation.

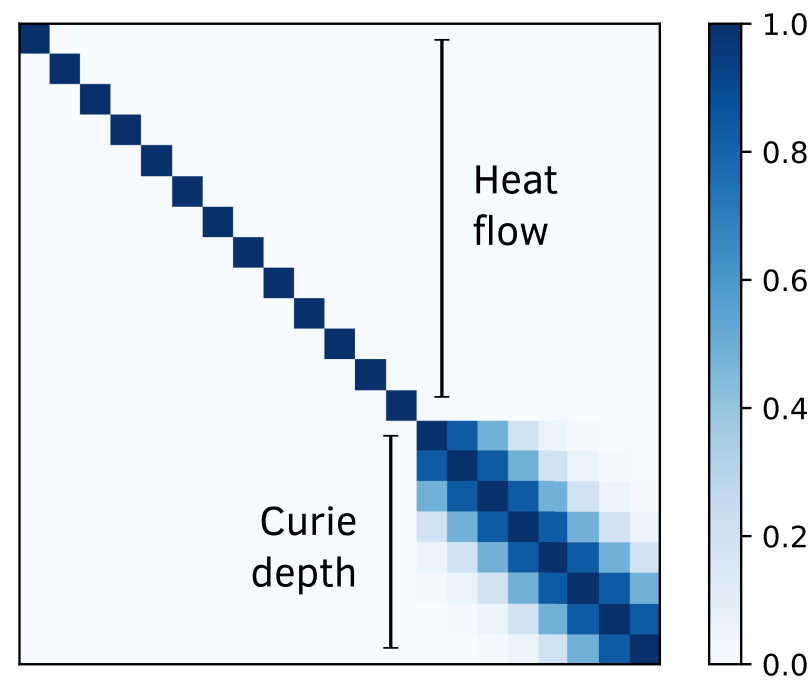

Figure 3. Normalized data covariance matrix, $\mathbf{C}_{d}$, with uncorrelated uncertainties for $\sigma_{q_{s}}$ and a Gaussian covariance function for $\sigma_{z_{580}}$, where the correlation length is related to the window size. The matrix is normalized by $\sigma^{2}$ for illustrative purposes.

Table 1. Priors for lithology-dependent thermal properties $(k, H)$ based on aggregated measurements from rock samples. All prior probabilities are normally distributed with $\sigma_{p}$ equal to one standard deviation. Units for $k, H$ and $q_{0}$ are $\mathrm{W} \mathrm{m}^{-1} \mathrm{~K}^{-1}, \mu \mathrm{W} \mathrm{m} \mathrm{m}^{-3}$ and $\mathrm{mW} \mathrm{m}^{-2}$, respectively.

\begin{tabular}{lcccc}
\hline & \multicolumn{4}{c}{ Priors $\left(\mathbf{m}_{p}, \sigma_{p}\right)$} \\
\cline { 2 - 5 } Lithology & $k$ & $\sigma_{k}$ & $H$ & $\sigma_{H}$ \\
\hline Moralana Supergroup & 3.11 & 0.45 & 2.41 & 1.05 \\
Nargoon Group & 3.50 & 0.26 & 1.46 & 1.06 \\
Castlemaine Group & 3.50 & 1.00 & 2.39 & 0.63 \\
St Arnaud Group & 3.66 & 0.60 & 2.28 & 0.42 \\
Costerfield Siltstone & 3.36 & 0.90 & 2.26 & 0.56 \\
Adminaby Group & 3.36 & 0.90 & 2.26 & 0.56 \\
Ordovician Shale & 3.40 & 0.60 & 2.63 & 0.10 \\
Cobbannah Group & 3.40 & 0.60 & 2.63 & 0.10 \\
Stavely Volcanics & 3.34 & 0.44 & 0.70 & 0.11 \\
Magdala Volcanics & 2.89 & 0.28 & 0.07 & 0.01 \\
Delamerian Proterozoic & 3.62 & 0.76 & 2.23 & 1.10 \\
\hline Selwyn Block & 3.64 & 0.59 & 1.85 & 0.93 \\
Lower flux boundary & $q_{0}=30.00$ & $\sigma_{q_{0}}=5.00$ \\
\hline
\end{tabular}

after which, $\mathrm{d} \mathbf{H}, \mathrm{d} q_{0}$, are unpacked from the vector $\mathrm{d} \mathbf{b}$.

To extract dk, we require the derivatives of $\mathbf{A}^{-1}$ from eq. (11), which we cannot evaluate directly. However, while $\mathbf{A}$ is differentiable and non-singular, we can use the relationship

$\frac{\mathrm{d} \mathbf{A}^{-1}}{\mathrm{~d} \mathbf{k}}=-\mathbf{A}^{-1} \frac{\mathrm{d} \mathbf{A}}{\mathrm{d} \mathbf{k}} \mathbf{A}^{-1}$,

which is described in the Appendix. Applying this to eq. (11) and rearranging terms gives,

$\mathrm{d} \mathbf{T}=\mathbf{A}^{-1} \mathrm{~d} \mathbf{b}-\mathbf{A}^{-1} \mathrm{~d} \mathbf{A} \cdot \mathbf{A}^{-1} \mathbf{b}$,

$\mathrm{d} \mathbf{T}$ can be evaluated from the solution of two systems of equations, $\Psi_{1}$ and $\Psi_{2}$,

$\mathbf{A} \Psi_{1}=\mathrm{d} \mathbf{b}$

$\mathbf{A} \Psi_{2}=\mathbf{X}$

where $\mathbf{X}=-\mathbf{A}^{-1} \mathrm{~d} \mathbf{A} \cdot \mathbf{A}^{-1} \mathbf{b}$ and $\mathrm{d} \mathbf{T}$ is simply the sum,

$\mathrm{d} \mathbf{T}=\Psi_{1}+\Psi_{2}$.

Matrix A contains all the linear conductivity terms where each element of the matrix is made up of second order finite difference terms with respect to $\mathbf{k}$. The derivative of $\mathbf{A}$ with respect to each element, $i$, in $\mathbf{k}$ is found using the chain rule,

$\mathrm{d} \mathbf{A}=\frac{\mathrm{d} \mathbf{A}}{\mathrm{d} k_{i}} \mathrm{~d} k_{i}$.

Substituting eq. (18) for $\mathrm{d} \mathbf{A}$ in eq. (17) forms an expression where the change in temperature with respect to $\mathbf{k}$ is the derivative of matrix A with respect to each element, $k_{i}$,

$\mathrm{d} \mathbf{T}=\mathrm{d} k_{i} \cdot \mathbf{X}=\mathrm{d} k_{i} \cdot\left(-\mathbf{A}^{-1} \frac{\mathrm{d} \mathbf{A}}{\mathrm{d} k_{i}} \mathbf{A}^{-1} \mathbf{b}\right)$,

where $\mathbf{X}=-\mathbf{A}^{-1} \mathrm{~d} \mathbf{A} / \mathrm{d} k_{i} \mathbf{A}^{-1} \mathbf{b}$ is a vector that does not change with T.

Now that we have obtained an expression linking $\mathrm{d} \mathbf{T}$ to $\mathrm{d} k_{i}$, we can obtain the adjoint, which is the sensitivity of $\mathbf{k}$ with respect to the objective function for each element, $i$. The adjoint for $\mathrm{d} \mathbf{T}=\mathrm{d} k_{i} \cdot \mathbf{X}$ 

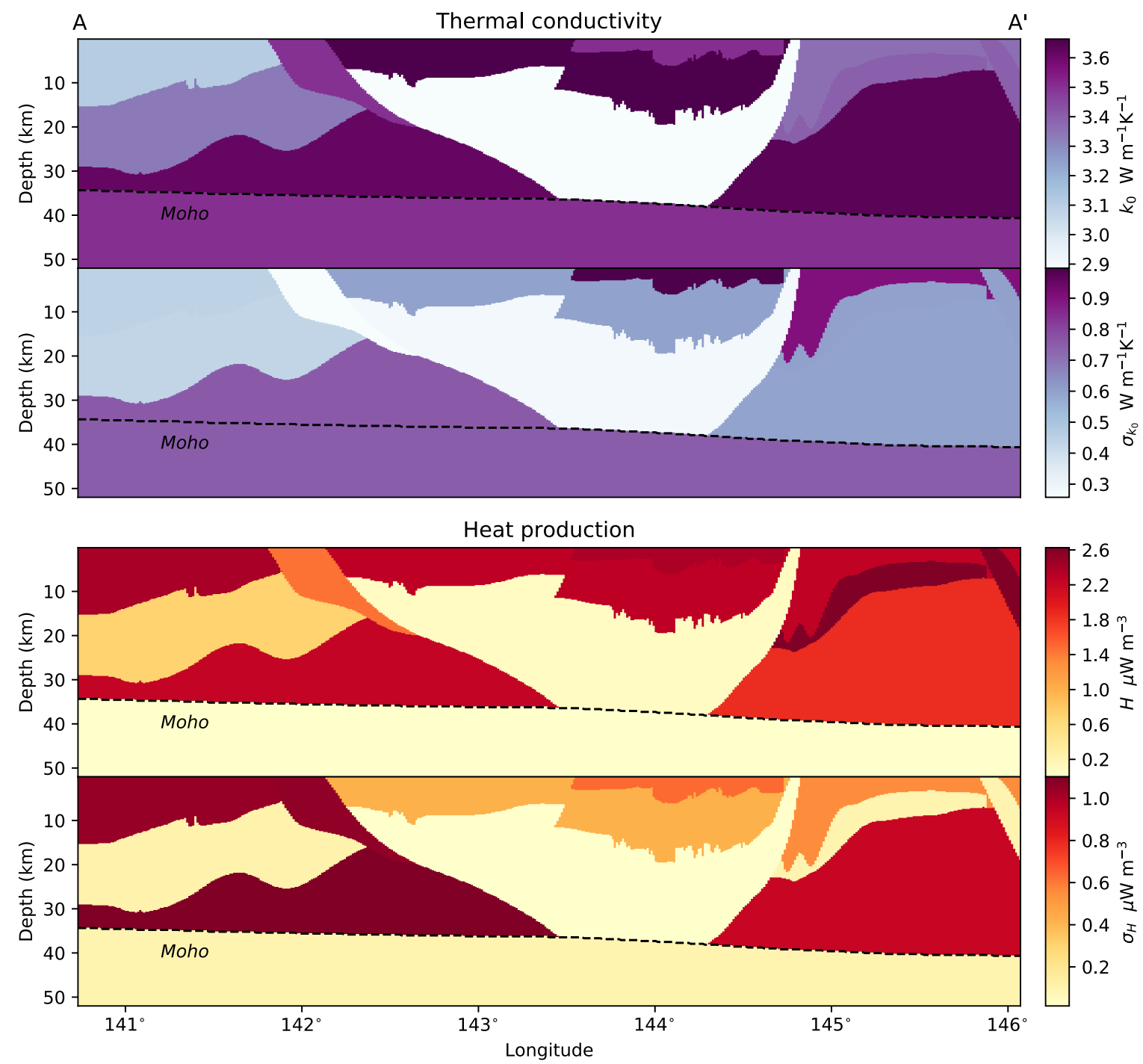

Figure 4. Lithology-dependent prior estimates of $k$ and $H$ mapped from Table 1 to the mesh. Errors are normally distributed where $\sigma_{k}$ and $\sigma_{H}$ are one equal to standard deviation from the mean.

is,

$\mathrm{d} k_{i}=\mathrm{d} \mathbf{T} \cdot \mathbf{X}=\mathrm{d} \mathbf{T} \cdot\left(-\mathbf{A}^{-1} \frac{\mathrm{d} \mathbf{A}}{\mathrm{d} k_{i}} \mathbf{A}^{-1} \mathbf{b}\right)$

At this point two computational savings are apparent: we have already stored $\mathbf{T}$ from evaluating the forward model, and we are interested only in the derivatives of $\mathbf{k}$ with respect to each lithology, $\mathrm{dk}_{L}$ (i.e. a region of the mesh that corresponds to a lithology $L$ ). Eq. (20) simplifies to

$\mathrm{d} \mathbf{k}_{L}=\mathrm{d} \mathbf{T} \cdot-\mathbf{A}^{-1} \frac{\mathrm{d} \mathbf{A}}{\mathrm{d} \mathbf{k}_{L}} \mathbf{T}$.

Now the number of linear solves correspond to the number of lithologies, $n$, where $\mathrm{d} \mathbf{A} / \mathrm{dk}_{L}$ is a constant matrix that can be stored for multiple adjoint evaluations since the geometry of each lithology is fixed. The field variable, $\mathrm{dk}$, can be found by summing the contribution from each lithology,

$\mathrm{d} \mathbf{k}=\sum_{L=1}^{n} \mathrm{~d} \mathbf{k}_{L}$.
Lastly, the field variables $\mathrm{d} \mathbf{k}$ and $\mathrm{d} \mathbf{H}$ (collected from eq. 12) are summed over each lithology, $L$,

$\mathrm{d} f: \sum_{i=1}^{L} \Omega_{i} \rightarrow \mathrm{d} \mathbf{m}$,

where $\mathbf{d} \mathbf{m}$ is equivalent to the input vector $\mathbf{m}$ used at the start of the forward model, except that it contains the gradient components of each parameter, $\mathrm{d} \mathbf{m}:\left[\mathrm{d} k_{1}, \mathrm{~d} k_{2}, \ldots, \mathrm{d} k_{n}, \mathrm{~d} H_{1}, \mathrm{~d} H_{2}, \ldots, \mathrm{d} H_{n}, \mathrm{~d} q_{0}\right]$. It is this vector, also written as $\nabla S(\mathbf{m})$, that we use to find the model of minimum misfit.

More sophisticated algorithms than the steepest-descent approach (eq. 9) evaluate the second derivative (Hessian) to find the curvature of $S(\mathbf{m})$, which improves rates of convergence for nonlinear problems. Newton's method stores the full Hessian matrix, which is very expensive to compute, whereas quasi-Newton methods replace the exact Hessian with an approximation found by successive evaluations of $\nabla S(\mathbf{m})$. We use a truncated Newton method for optimization constrained within parameter limits (Nash 1984, 2000), which stores an implicit approximation of the Hessian with only a few vectors. Convergence is reached when either $\left|\mathbf{m}_{n}-\mathbf{m}_{n-1}\right|<1 \times 10^{-12}$ or $\left|S\left(\mathbf{m}_{n}\right)-S\left(\mathbf{m}_{n-1}\right)\right|<1 \times 10^{-12}$. 


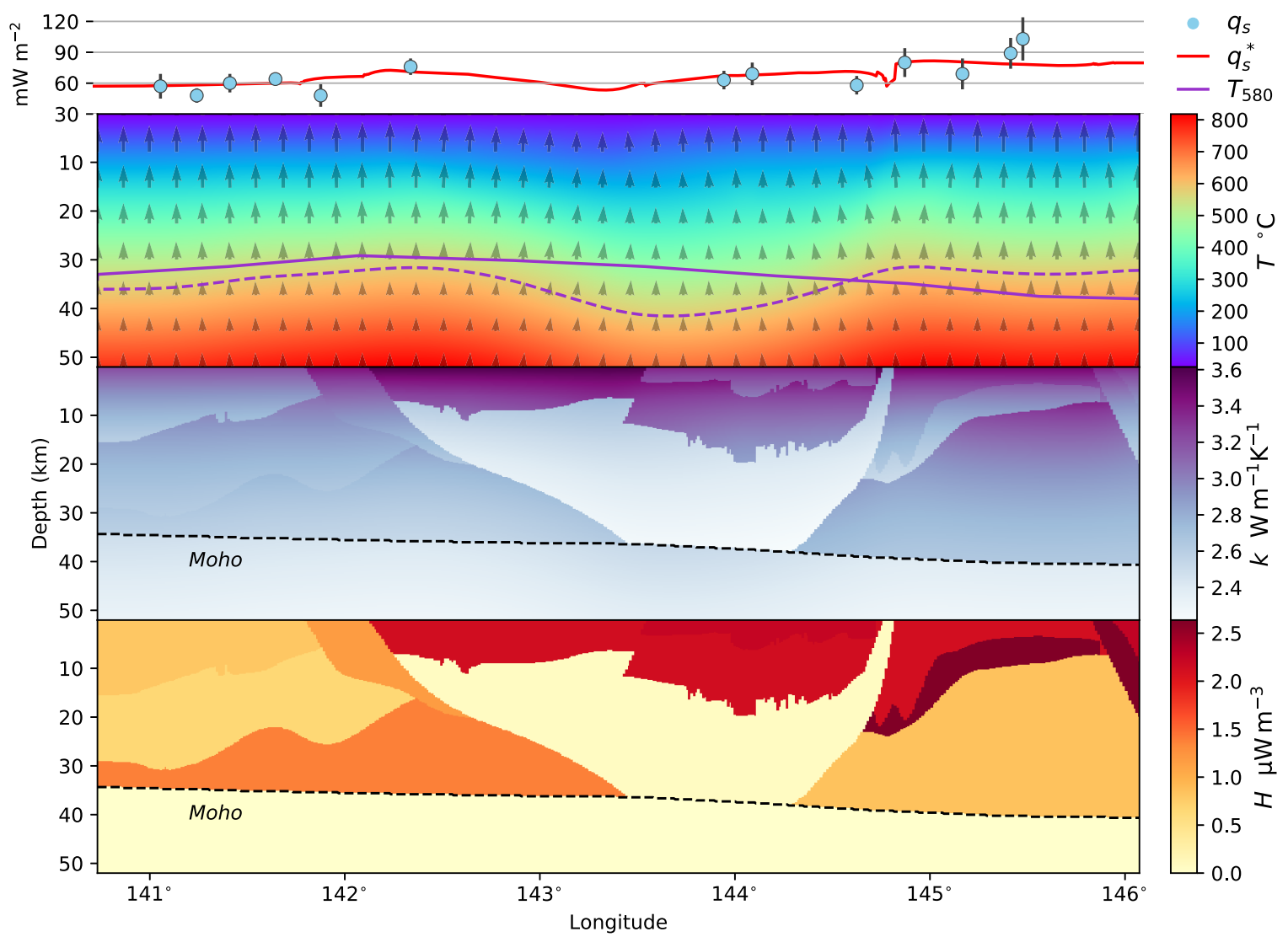

Figure 5. Model of minimum misfit given a priori information in Table 1 and constrained by the Curie depth and surface heat flow data $\left(z\left(T_{580}\right)\right.$ and $\left.q_{s}\right)$. The top pane shows the temperature distribution with the modelled surface heat flow $\left(q_{s}^{*}\right)$ compared against the data $\left(q_{s}\right)$. The purple lines indicate the Curie depth (dashed: simulated; solid: data). Effective thermal conductivity, $k(T)$, and heat production of the crust are visualized in the bottom two panes.

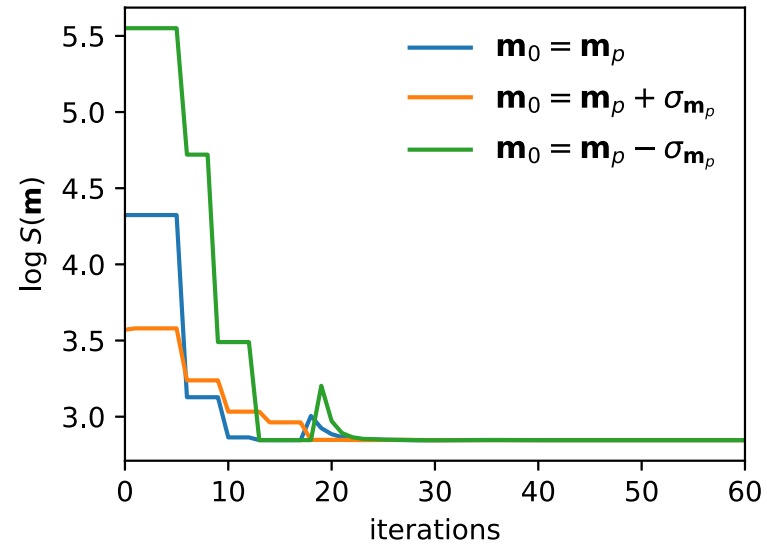

Figure 6. Inversions were initiated at different starting points, $\mathbf{m}_{0}$, related to their priors in Table 1. All inversions converge to an identical model of minimum misfit in approximately the same number of iterations.

\subsection{Non-uniqueness}

While the MAP estimate can be found very efficiently using the adjoint, there may be a number of models that fit the Curie depth and heat flow data for many combinations of model parameters. From eq. (4), any nonlinearity in $\mathbf{g}$ causes the posterior probability distribution to depart from the simple Gaussian form assumed in measurement errors and introduces the possibility of multiple minima in $S(\mathbf{m})$. The steady-state thermal diffusion problem becomes

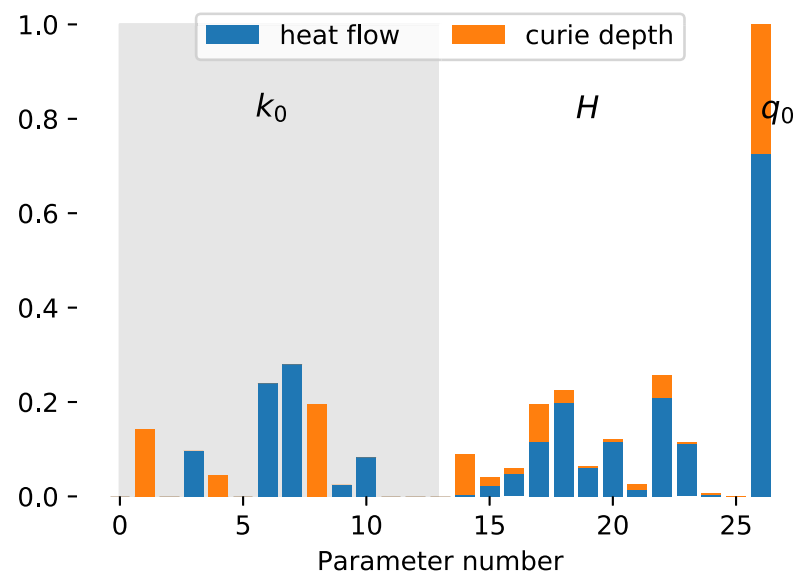

Figure 7. Normalized sensitivity of the objective function to different inversion variables $\left(k, H, a\right.$, and $\left.q_{0}\right)$. The total sensitivity for each parameter has been divided into two components that constrain the misfit $\mathbf{g}(\mathbf{m})-\mathbf{d}$ in the objective function-heat flow and the Curie depth.

nonlinear due to the dependence of thermal conductivity on temperature (eq. 6). Gradient methods are local optimization techniques and can become trapped by a local minimum. This can be avoided if the gradient search can be initiated as close to the MAP estimate as possible, or if multiple inversions are initiated from different starting positions. We couple gradient methods with Monte Carlo sampling to address the problem of identifying multiple minima in the parameter space and identify parameters which are non-unique. This 


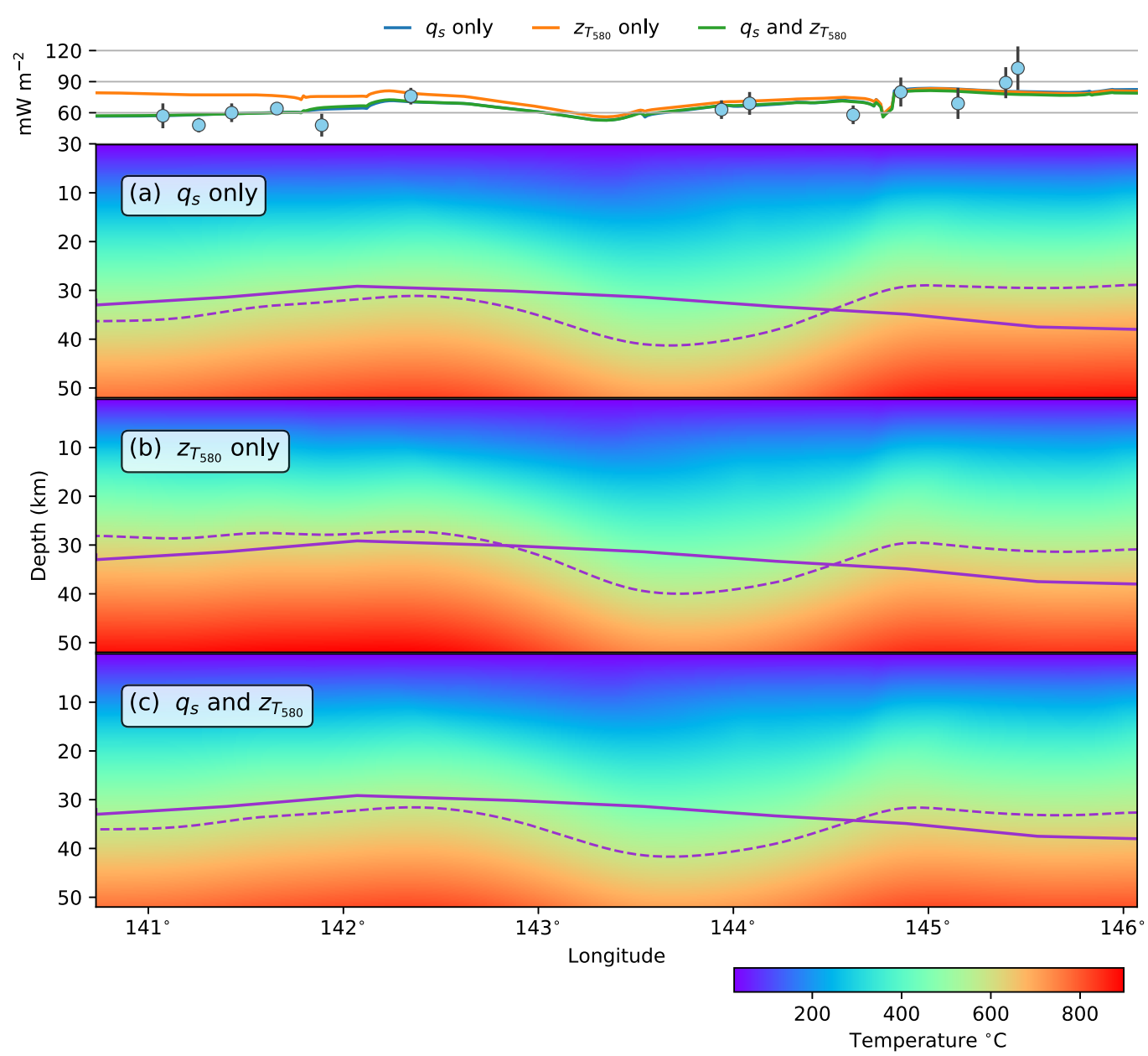

Figure 8. Model of minimum misfit assimilating (a) heat flow data only $\left(q_{s}\right)$, (b) the Curie depth only $\left(z_{T_{580}}\right)$ and (c) heat flow and the Curie depth ( $q_{s}$ and $z_{T_{580}}$ ). The purple line is the Curie isotherm (dashed: simulated; solid: data). Priors are identical between all three simulations (Table 1).

strategy produces an approximation to the posterior by randomly perturbing prior information within their probability distribution to build an ensemble of inversions, from which we estimate uncertainty and covariance information.

\section{MODEL LAYOUT OF SOUTHEASTERN AUSTR A L I A}

Southeastern Australia is an ideal study area to apply the inversion framework presented above due to the dense sampling of different geophysical data, and a well resolved geological model (Fig. 1). We implement a 2-D solver to demonstrate the adjoint we have formulated in the previous section. The dominant structural grain is oriented N-S in SE Australia, thus we choose an E-W cross-section through $\mathbf{A}$ to $\mathbf{A}^{\prime}$ (Fig. 2) that intersects most of the major lithologies and follows a seismic transect that has previously been interpreted (Cayley et al. 2011).

The crust in Southeast Australia is composed of Precambrian lower crustal rocks, Cambrian mafic volcanics and OrdovicianSilurian metasediments in the upper crust. The east-dipping Moyston fault offsets the Delamerian Orogen and the Lachlan Orogen which have distinct thermal regimes (Mather et al. 2017). We invert the values of $k$ and $H$ for 11 lithologies present in the cross-section, and the lower flux boundary condition, $q_{0}$ to characterize the thermal structure in Southeast Australia. This equates to a total of 23 variables (i.e. the size of vector $\mathbf{m}$ is 23 ) that are constrained by surface heat flow data and the depth to Curie temperature.

\subsection{Curie depth and heat flow data}

Two independent data types related to temperature are assimilated: surface heat flow and depth to the Curie temperature. The Curie temperature is the point at which magnetic sources lose their permanent magnetic properties. The dominant magnetic mineral in the crust is magnetite, which has a Curie temperature of $580{ }^{\circ} \mathrm{C}$, and the depth to the Curie temperature is often interpreted to be the depth to $580{ }^{\circ} \mathrm{C}$ isotherm. This presents a useful isotherm to constrain the lower crust, however there is some uncertainty as other magnetic minerals may be present that have different Curie temperatures. Furthermore, methods to determine the Curie depth are highly sensitive to the window size of the magnetic anomaly chosen by the interpreter. We incorporate the Australian compilation of Curie depth determinations from Chopping \& Kennett (2015), where a $400 \times 400 \mathrm{~km}$ moving window of the magnetic anomaly was used to compute the Curie depth with a centroid spacing of $60 \mathrm{~km}$. These Curie depth determinations are therefore correlated within this $400 \mathrm{~km}$ wavelength and we accordingly choose a Gaussian function to parametrize the covariance matrix,

$\mathbf{C}\left(\mathbf{x}, \mathbf{x}^{\prime}\right)=\sigma^{2} \exp \left(-\frac{\left\|\mathbf{x}-\mathbf{x}^{\prime}\right\|^{2}}{2 h^{2}}\right)$, 


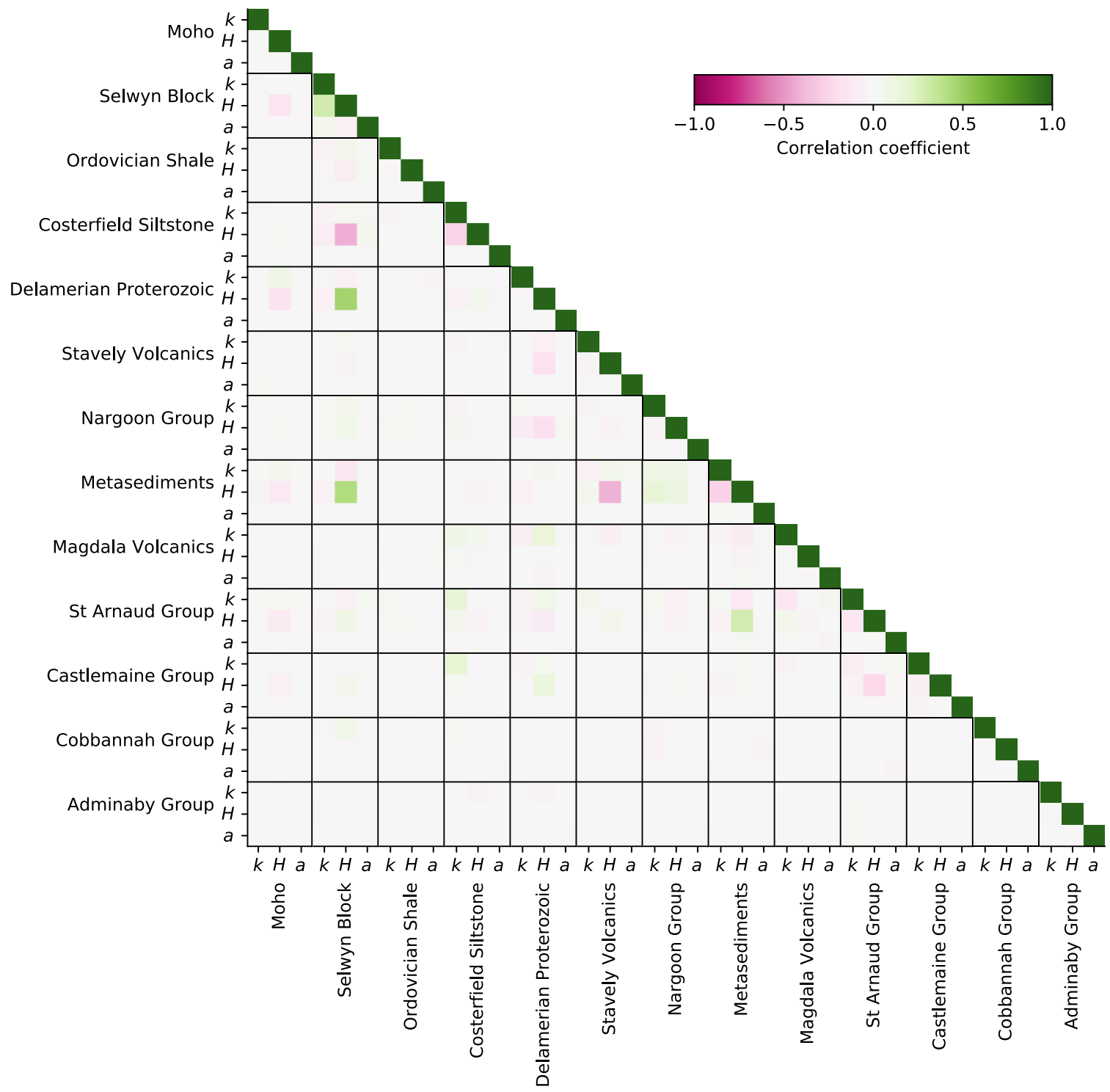

Figure 9. Correlation coefficient matrix for lithology-related variables $(k, H, a)$ in Southeastern Australia.

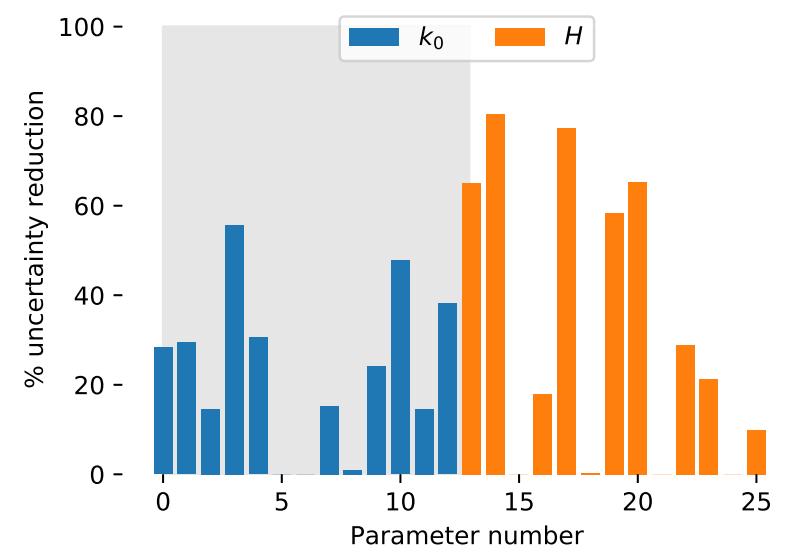

Figure 10. Posterior uncertainty reduction compared to the prior uncertainty due to constraints in the cost function.

where $\left\|\mathbf{x}-\mathbf{x}^{\prime}\right\|$ is Euclidean distance between each point, and $h$ is the correlation length scale. Alternatively, each surface heat flow data point is an independent observation of the thermal field and are thus uncorrelated. $13 q_{s}$ data points within $10 \mathrm{~km}$ of the crosssection are incorporated in our inversions from the compilation of Mather et al. (2017). Their probabilities are normally distributed with uncertainties attributed to fluid flow in aquifers, imprecise thermal conductivity measurements on core samples, or the method used to calculate surface heat flow, amongst others. Our data covariance matrix $\mathbf{C}_{d}$ is sparse for heat flow data (the same non-zeros as an identity matrix) and significantly denser for the Curie depth (Fig. 3). The inverse covariance matrix $\mathbf{C}_{d}^{-1}$ is never explicitly evaluated, rather we solve as a system of equations every time the objective function is computed: $\mathbf{C}_{d} \Theta=\mathbf{g}(\mathbf{m})-\mathbf{d}$, where $\mathbf{C}_{d}$ is a constant matrix that can be stored for all evaluations.

\subsection{A priori thermal properties}

The thermal conductivity and heat production of various lithologies have been constrained from measurements on core samples and rock outcrops. These form the prior information for our inversions (Table 1) which are mapped to the Cartesian domain, $\Omega$ 


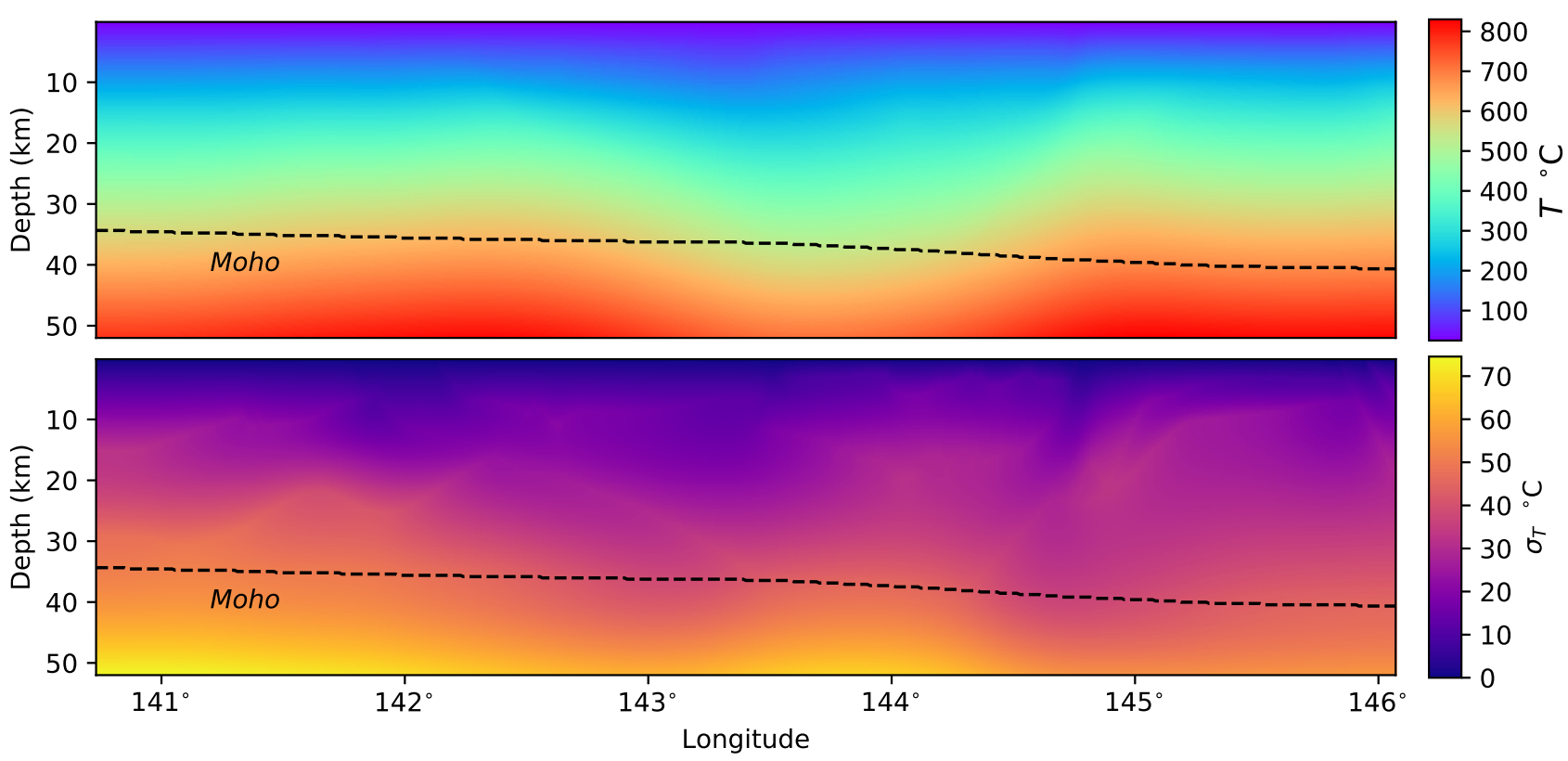

Figure 11. Mean temperature distribution from an ensemble of model realizations (upper pane); standard deviation, $\sigma_{T}$ of the ensemble (lower pane).

(Fig. 4). Uncertainty was calculated by the propagation of errors from laboratory measurements and the amount of variation within a single lithology. Lithologies with the lowest uncertainty is the Cambrian mafic volcanics followed by the andesitic Stavely Volcanics. The Selwyn Block and Proterozoic crust of Delamerian origin are most uncertain because they are comprised of multiple smaller rock formations of varying compositions. The uncertainty of these lithology-dependent priors are uncorrelated, which means the $\ell_{2}$-norm objective function may be used.

\section{RESULTS AND DISCUSSION}

The MAP estimate combining both $q_{s}$ and $z_{T_{580}}$ was obtained in 67 adjoint evaluations, which is at least one order of magnitude less than a Markov-Chain Monte Carlo (MCMC) simulation would generally require of the forward model. The simulated temperature field suggests that the distribution of temperature in Southeast Australia is systematically hotter within Precambrian crust, and cooler in the Cambrian mafic volcanics (Fig. 5). Proterozoic crust of Delamerian origin contains high rates of heat production, which raises temperatures in the lower crust. It is unusual for the lower crust to be so highly enriched in heat-producing elements: values of $\sim 0.5 \mu \mathrm{W} \mathrm{m}^{-3}$ are considered normal for the lower crust because melt extraction and tectonism are believed to rework radiogenic elements into the upper crust, irreversibly stabilising the continental lithosphere (Sandiford \& McLaren 2002). The Tasman Orocline closed in the Early Devonian (Moresi et al. 2014), which does not preclude its stability, but may facilitate the incorporation of a radiogenic lower crust in this dominantly Phanerozoic crustal composition.

\subsection{Parameter sensitivity}

We initiated inversions at three different starting positions, $\mathbf{m}_{0}$, related to the prior information, and all converge to the MAP estimate in approximately the same number of iterations (Fig. 6). The sensitivity of the objective function to the inversion variables is determined by examining the structure of the Jacobian matrix $\mathbf{J}(\mathbf{m})$. As the Jacobian is related to the gradient by $\nabla S\left(\mathbf{m}_{i}\right)=\mathbf{J}\left(\mathbf{m}_{i}\right)^{\mathrm{T}} \mathbf{g}\left(\mathbf{m}_{i}\right)$, we can find the sensitivity of the objective function with respect to each parameter from the gradient vector at the model of minimum misfit, $S\left(\mathbf{m}_{n}\right)$. We separately examine the sensitivity of the Curie depth and heat flow data to the inversion variables to enable a comparison of the constraints each data set brings to the thermal structure of the crust (Fig. 7). For inversions that assimilate only surface heat flow, the objective function is relatively insensitive to $k$ compared to $H$ and $q_{0}$. This is expected because $q_{0}$ is by far the most spatially pervasive variable that we invert. Alternatively, for a model constrained only by the Curie depth, the strongest sensitivities to $k$ come from surface lithologies that intersect $q_{s}$ and lithologies that cross the Curie isotherm. A combination of these two data sets are required to resolve the model parameters: heat flow data constrain the integrated heat flux beneath each point of measurement (Fig. 8a), and the Curie depth provides an important constraint to lower crustal temperatures (Fig. 8b). Omission of either data set results in a problem that is ill-posed.

\section{POSTERIOR ANALYSIS}

Gradient-based inversion returns the MAP estimate for a configuration of a priori information on model parameters and data constraints. However, quantifying the posterior uncertainty and covariance relationships can be accomplished by perturbing priors within their probability density functions, using Monte Carlo sampling, to build an ensemble of model realizations. After many simulations, we assemble a posterior distribution that samples the posterior probability density function (Fisher 2003; Chevallier et al. 2007).

\subsection{Parameter covariance}

Vertically stacked lithologies are negatively correlated since a decrease in heat production of one layer will be compensated in the other match surface heat flow. The strongest negative covariance is observed between the rates of heat production within the Selwyn 
Block and Costerfield Siltstone, and also between the Stavely Volcanics and Moralana Supergroup (Fig. 9). These covariances reflect the thermal budget in $1-\mathrm{D}, q_{s}=\int_{z} H+q_{0}$, where mantle heat flow and heat sources integrate to $q_{s}$. Positive covariances are observed between the Selwyn Block and Delamerian Proterozoic crust as a result of the spatial covariance relationship within the Curie depth data set. We observe positive covariance between lithologies that intersect this $580^{\circ} \mathrm{C}$ isotherm because the uncertainties of $z_{T_{580}}$ are smeared across the lower crust from $\mathbf{C}_{d}$.

The amount of precision gained by the posterior relative to the prior is denoted $\delta \sigma$,

$\delta \sigma=1-\frac{\sigma_{\text {posterior }}}{\sigma_{\text {prior }}}$

where $0 \leq \delta \sigma<1$. Here, the posterior uncertainty reduction is most significant in rates of heat production (Fig. 10), which shows that Curie depth and heat flow data have greatly improved the precision of these variables. In particular, the reduction in $\sigma_{H}$ of the Selywn Block is 80 per cent relative to the prior. Some improvement is also notable in the thermal conductivity of lithologies that intersect heat flow observations and the Curie isotherm. The posterior probability for $q_{0}$ remains unchanged from its prior distribution despite the strong sensitivity of the objective function to this variable. Negative covariance was observed between $q_{0}$ with rates of heat production in overlying layers and suggests the sensitivity of the objective function to $q_{0}$ is confounded by similar sensitivities to $H$ for overlying lithologies so that strong posterior relationships are established among these variable, but individual uncertainties are hardly reduced.

The uncertainty reduction for each parameter translates to more precise estimates of temperature at depth. The mean and standard deviation of all temperature solutions in the ensemble is presented in Fig. 11 (The mean of the ensemble is identical to the MAP estimate we found in Fig. 5.) Uncertainty increases with depth until a maximum of $\sigma_{T}=75^{\circ} \mathrm{C}$ is reached beneath Delamerian Proterzoic crust. This is primarily controlled by the rate of heat production within this lithology, which has a posterior uncertainty of $\sigma_{H}=0.3 \mu \mathrm{W} \mathrm{m}{ }^{-3}$. This is reduced from its a priori uncertainty of $1.1 \mu \mathrm{W} \mathrm{m}^{-3}$, due to its strong control on lower crustal temperatures in this region of the model domain. Temperature variation above the Moho is well constrained with uncertainties not exceeding $50^{\circ} \mathrm{C}$ and illustrates that, for a fixed geometry, precise models of temperature variation in the crust are obtained by assimilating Curie depth and surface heat flow data.

\section{RELAXING GEOLOGICAL CONSTRAINTS}

Up to this point we have inverted thermal properties assigned to a rigid geological / lithological model of the crust. In this section we relax the geological restriction imposed on this model by introducing new inversion variables that accommodate geological uncertainty. Our inverse problem remains identical to those formulated in Section 2, except we invert thermal conductivity and rates of heat production for each node in the mesh. Our mesh size is $800 \times 248$, which corresponds to 396800 inversion variables. An inverse problem of this size is only possible because of the efficiency of the adjoint method at minimizing high dimensional functionals. The solution is, however, strongly dependent on the choice of starting model because a large combination of parameters can satisfy our thermal constraints. We introduce a prior covariance matrix to mitigate this problem.

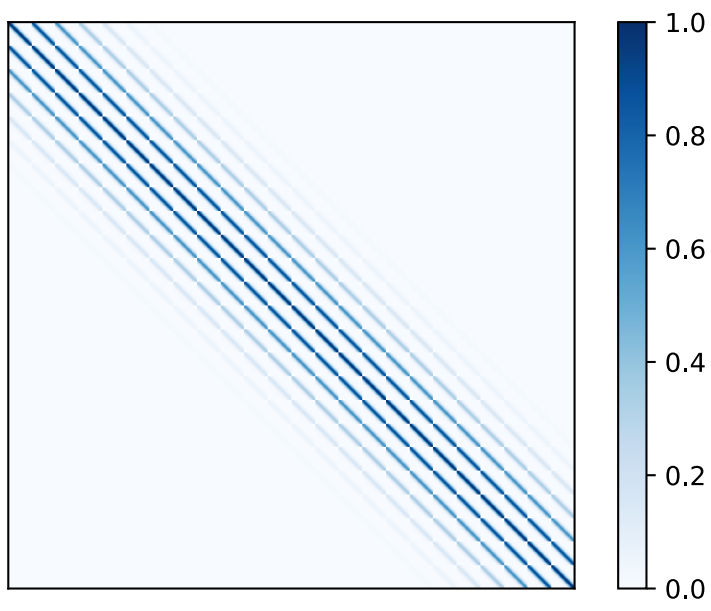

Figure 12. Normalized prior covariance matrix, $\mathbf{C}_{p}$, for whole-mesh inversions. Note that the mesh has been down-sampled from $800 \times 248$ to $80 \times 24$ and normalized by $\sigma_{p}^{2}$ for visual clarity.

\subsection{Prior covariance}

The continuity of thermal properties beyond boundaries imposed by a geological model is a physical possibility that we explore. From a geological standpoint, the rheology of one point in a rock package is relatively similar to the points surrounding it, which we parametrize using a prior covariance matrix, $\mathbf{C}_{p}$ (Fig. 12). This is a banded diagonal matrix with a correlation length scale, $h$, related to the distance between adjacent nodes on the mesh, and uncertainties set to those in Table 1 (visualized in Fig. 4). As $h$ increases, so does the number of off-diagonal elements in $\mathbf{C}_{p}$. Increasing the correlation length produces a similar effect to a Gaussian smoothing filter, where the influence of each inversion variable is smeared across the mesh at increasing radii. We initiate the inversion using $h=1 \mathrm{~km}$. This drastically reduces the prevalence of solutions that locally alter a value of thermal conductivity or heat production at each data point to lower the misfit.

\subsection{MAP estimate}

We initiated the inversion at the previous MAP estimate (Fig. 13) until convergence was reached after 2167 evaluation of the adjoint model. This is close to a linear increase with the number of inversion variables and compares with MCMC methods where the number of iterations to find the MAP scales exponentially with unknown variables.

The simulation fits Curie depth and heat flow data better than the geometrically constrained version because thermal conductivity and rates of heat production at each node in the mesh are separate inversion variables (Fig. 13). The Moho in our model has been shifted upwards by $2 \mathrm{~km}$ to match the Curie depth on the western half of the domain. Rates of heat production are predicted to be slightly higher in the new model within the Cambrian mafic volcanics and decreased in the Selwyn Block to complement the long wavelength variation in Curie depth. The fit to surface heat flow data has remained unchanged largely because the correlation length prohibits spurious values from arising locally. It is not immediately intuitive that Curie depth should appear to be weighted more than heat flow data within the inversion framework. However, in order to match all heat flow data, the local alteration to the heat production field results in high misfit, which is compounded by the spatial correlation in neighbouring nodes on the mesh. In comparison, a 


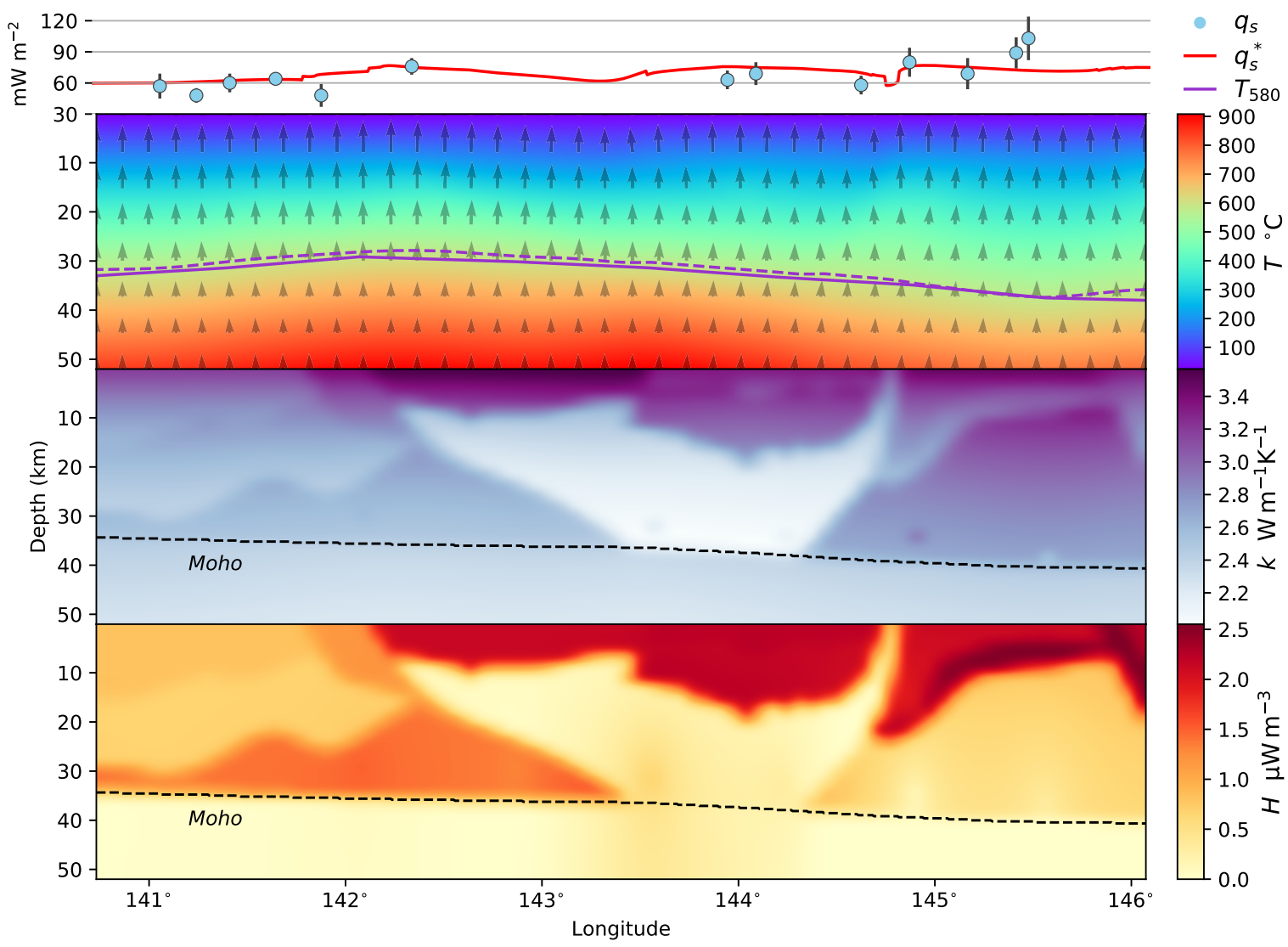

Figure 13. Model of minimum misfit constrained by the Curie depth and surface heat flow data $\left(z_{T_{5} 80}\right.$ and $\left.q_{s}\right)$ without a priori geological restriction. Variation in the $k(T)$ and $H$ occur at long wavelengths due to spatially correlated uncertainties in $\mathbf{C}_{p}$.

better fit to Curie depth can be achieved with a minor adjustment of thermal conductivity and heat production at the Moho albeit with a relatively small change in misfit.

If prior uncertainties are uncorrelated (as $h \rightarrow 0$ ) then values of heat production and thermal conductivity are altered at the node immediately adjacent to a heat flow data point. The smoothness we have imposed on the variation of thermal properties, as a consequence of the prior covariance matrix, may be undesirable because lithologies are generally separated by sharp discontinuities. However, the approach we have taken here is useful to refine an existing geological model to better fit the data.

\section{LIMITATIONS AND FUTURE DIRECTIONS}

The above sections demonstrate the role of jointly assimilating Curie depth and heat flow data on the thermal structure of the crust. For a prescribed geological model, populated with correlated and uncorrelated prior information on rates of heat production and thermal conductivity, we found the MAP estimate of temperature subject to the data we assimilated (Figs 5 and 13). However, Curie depth has high intrinsic uncertainty related to the choice of fractal parameter and size of the window used to discretize maps of the magnetic anomaly (Audet \& Gosselin 2019; Mather \& Fullea 2019), and heat flow data are occasionally perturbed by hydrothermal circulation and suffer from low spatial coverage (Mather et al. 2017). These data loosely constrain the $580{ }^{\circ} \mathrm{C}$ isotherm and the integrated heat production in sparsely distributed localities for Curie depth and heat flow data, respectively. Additional constraints from seismic velocity could further reduce the uncertainty of subsurface temperature models. In addition, the geological model may be refined as a consequence of assimilating new data sets to introduce additional lithologies that are unresolved by the current data.

Despite these shortcomings of the constraining data, our gradientbased method to solve the inverse problem of heat conduction is highly applicable to many geological contexts where multiple data types sensitive to temperature are available. Due to the steady-state limitation of our solver, stable tectonic locations are particularly well suited where secondary processes such as hydrothermal advection do not impart a significant role on the thermal regime. The high convergence rate and low computation time of this method to find the optimal temperature model, subject to data and prior information, renders it suitable to real-time analysis of subsurface thermal structure for a modest set of inversion variables. Note that, as with all inverse problems, the convergence rate will decrease with the number of variables. If, for example, we introduce a variable heat flux boundary condition at the base of our model, which may be required in certain terranes, then the computation time will increase due to a higher number of variables. Likewise, mapping constant thermal properties to discreet lithologies on the mesh dramatically decreases the inversion variables and computation time required to invert the thermal regime.

\section{CONCLUSIONS}

Using a geological model of SE Australia, we estimate the optimal configuration of thermal conductivity and rates of heat production 
that best reproduce Curie depth and surface heat flow data. We determine that temperatures are highest beneath Precambrian lithologies in our model, with Moho temperatures exceeding $650{ }^{\circ} \mathrm{C}$ due to high rates of heat production $\left(0.9-1.4 \mu \mathrm{W} \mathrm{m}^{-3}\right)$. In comparison, the dominantly Phanerozoic portion of the crust reaches only $520{ }^{\circ} \mathrm{C}$ in Cambrian mafic volcanics, which highlights the division within this accretionary terrane. Quantifying these results with a sensitivity analysis, we observed strong negative covariances between the lower flux boundary condition, $q_{0}$, and rates of heat production in vertically stacked lithologies beneath heat flow data points. This stems from balancing the heat flow budget among various lithologies to reduce the misfit with surface heat flow data. The maximum uncertainty from our ensemble of temperature solutions is $73{ }^{\circ} \mathrm{C}$, which demonstrates that Curie depth and heat flow data provide precise constraints on the thermal structure of the crust. Omitting either data set results in parts of the model that are unconstrained. A better fit to these data is obtained by relaxing geological boundaries through the use of a prior covariance matrix; however, this comes at the expense of more iterations to locate the MAP estimate.

\section{ACKNOWLEDGEMENTS}

This work was supported by resources provided by the Pawsey Supercomputing Centre with funding from the Australian Government and the Government of Western Australia.

\section{REFERENCES}

Audet, P. \& Gosselin, J.M., 2019. Curie depth estimation from magnetic anomaly data: a re-assessment using multitaper spectral analysis and Bayesian inference, Geophys. J. Int., 218(1), 494-507.

Cammarano, F., Goes, S., Vacher, P. \& Giardini, D., 2003. Inferring uppermantle temperatures from seismic velocities, Phys. Earth planet. Inter., 138(3-4), 197-222.

Cayley, R.A. et al., 2011. Crustal architecture of central Victoria: results from the 2006 deep crustal reflection seismic survey, Aust. J. Earth Sci., 58(2), 113-156.

Chevallier, F., Bréon, F.-M. \& Rayner, P.J., 2007. The contribution of the orbiting carbon observatory to the estimation of $\mathrm{CO}_{2}$ sources and sinks: theoretical study in a variational data assimilation framework, J. geophys. Res., 112, D09307.

Chopping, R. \& Kennett, B.L., 2015. Maximum depth of magnetisation of Australia, its uncertainty, and implications for Curie depth, GeoResJ, 7, $70-77$.

Cull, J.P., O’Reilly, S.Y. \& Griffin, W.L., 1991. Xenolith geotherms and crustal models in Eastern Australia, Tectonophysics, 192(3-4), 359-366.

Faul, U. \& Jackson, I., 2005. The seismological signature of temperature and grain size variations in the upper mantle, Earth planet. Sci. Lett., 234(1-2), 119-134.

Fisher, M., 2003. Estimation of Entropy Reduction and Degrees of Freedom for Signal for Large Variational Analysis Systems, ecmwf techedn, European Centre for Medium-Range Weather Forecasts.

Goes, S., Simons, F.J. \& Yoshizawa, K., 2005. Seismic constraints on temperature of the Australian uppermost mantle, Earth planet. Sci. Lett., 236(1), 227-237.

Hofmeister, A., 1999. Mantle values of thermal conductivity and the geotherm from phonon lifetimes, Science, 283(5408), 1699-706.

Karato, S.-i., 2003. Mapping water content in the upper mantle, Geophys. Monogr. Ser., 138, 135-152.

Mather, B. \& Fullea, J., 2019. Constraining the geotherm beneath the British Isles from Bayesian inversion of Curie depth: integrated modelling of magnetic, geothermal, and seismic data, Solid Earth, 10(3), 839-850.
Mather, B., McLaren, S., Taylor, D., Roy, S. \& Moresi, L., 2017. Variations and controls on crustal thermal regimes in Southeastern Australia, Tectonophysics, 723, 261-276.

Moresi, L., Betts, P.G., Miller, M.S. \& Cayley, R.A., 2014. Dynamics of continental accretion, Nature, 508(7495), 245-248.

Mosegaard, K. \& Tarantola, A., 1995. Monte Carlo sampling of solutions to inverse problems, J. geophys. Res., 100(B7), 12 431-12 447.

Nash, S.G., 1984. Newton-type minimization via the Lanczos Method, SIAM J. Numer. Anal., 21(4), 770-788.

Nash, S.G., 2000. A survey of truncated-Newton methods, J. Comput. Appl. Math., 124(1-2), 45-59.

O’Reilly, S.Y. \& Griffin, W.L., 1985. A xenolith-derived geotherm for Southeastern Australia and its geophysical implications, Tectonophysics, 111(12), 41-63.

Rawling, T.J., Osborne, C.R., McLean, M.A., Skladzien, P.B., Cayley, R.A. \& Williams, B., 2011. 3D Victoria Final Report, in GeoScience Victoria 3D Victoria Report, Chap. 14, pp. 1-98, Department of Primary Industries.

Sandiford, M. \& McLaren, S., 2002. Tectonic feedback and the ordering of heat producing elements within the continental lithosphere, Earth planet. Sci. Lett., 204(1-2), 133-150.

Tarantola, A., 2005. Inverse Problem Theory and Methods for Model Parameter Estimation, Society for Industrial and Applied Mathematics.

\section{APPENDIX: DERIVATIVE OF AN INVERSE MATRIX}

The derivative of the inverse of a square matrix, A, can be accomplished without evaluating the inverse matrix directly. To prove eq. (13), suppose $a_{i j}(k)$ are component functions for $\mathbf{A}$, and $a^{j h}(k)$ are component functions for $\mathbf{A}^{-1}(k)$, then for each $k$ we have

$\sum_{j=1}^{n} a_{i, j}(k) a^{j, h}(k)=\delta_{i}^{h}$,

where $n$ is the order of $\mathbf{A}$ and $\delta_{i}^{h}$ is the Kronecker delta symbol; hence,

$\sum_{j=1}^{n} \frac{\mathrm{d} a_{i j}}{\mathrm{~d} k} a^{j h}+a_{i, j} \frac{\mathrm{d} a^{j h}}{\mathrm{~d} k}=0$.

Evaluating the sum of these components and rearranging the inverse terms give eq. (13). Another way to test this is to derive the equation of the identity matrix, I, with respect to its elements, $k$,

$\mathbf{I}=\mathbf{A} \mathbf{A}^{-1}$

$\frac{\mathrm{d}}{\mathrm{d} k} \mathbf{I}=\frac{\mathrm{d}}{\mathrm{d} k}\left(\mathbf{A A}^{-1}\right)$

$0=\frac{\mathrm{d} \mathbf{A}}{\mathrm{d} k} \mathbf{A}^{-1}+\mathbf{A} \frac{\mathrm{d} \mathbf{A}^{-1}}{\mathrm{~d} k}$

$\frac{\mathrm{d} \mathbf{A}^{-1}}{\mathrm{~d} k}=-\mathbf{A}^{-1} \frac{\mathrm{d} \mathbf{A}}{\mathrm{d} k} \mathbf{A}^{-1}$

The derivative of the identity matrix is zero, and we evaluate the derivative of $\mathbf{A}$ and $\mathbf{A}^{-1}$ using the product rule. Rearranging for $\mathrm{d} \mathbf{A}^{-1} / \mathrm{d} k$ returns the expression in eq. (13). 